\title{
Energy saving potentials of a centralized hybrid heating system via adaptive model predictive control in a Northern Italy residential building
}

\author{
Ettore Zanetti ${ }^{1}$, Rossano Scoccia ${ }^{1}$, Silvia Garone ${ }^{2}$, Marcello Aprile ${ }^{1}$, Mario Motta ${ }^{1}$, Livio \\ Mazzarella $^{1}$ \\ ${ }^{1}$ Department of energy, Politecnico di Milano, 20156 Milan, Italy \\ ${ }^{2}$ Enersem, 20133 Milano, Italy
}

\begin{abstract}
Extensive research has been conducted on advanced control techniques for buildings in recent years. However, even though in theory and in few experimental studies, the benefit of advanced Building Energy Management Systems was shown in most newly built or renovated buildings a traditional approach is still preferred, due to the higher cost and complexity of more advanced approaches despite the benefits. This paper presents a Python-Modelica grey-box modelling and adaptive optimal control of a hybrid heating system coupled with a thermal storage, that aims to reduce costs and improve the performance by better exploiting the hybrid heating system and the thermal storage, within the project TEPORE. The results show that for the chosen case study there are not enough degrees of freedom for the hybrid heating system to be optimized. However, interesting results emerged from changing the heating capacity of the generators showing on average $50 \%$ reduction in running costs.
\end{abstract}

\section{Introduction}

Reducing the carbon footprint is one of EU main objectives. Residential buildings impact heavily on the final energy consumption in the EU zone, accounting for $25 \%$ of the total final energy consumption, $64 \%$ of which goes to the heating system (Eurostat, 2016). Therefore, reducing their consumption has been one of the primary goals of $\mathrm{H} 2020$ initiatives and EU policies.

Thanks to these funds a lot of research has been carried out on advanced control techniques for buildings in recent years (Thieblemont et al., 2017). However, even though in theory (Afram and Janabi-Sharifi, 2014) and in few experimental studies (De Coninck and Helsen, 2016), the benefit of advanced Building Energy Management Systems (BEMS) was shown, a lot of newly built and renovated buildings still lack the implementation of these advanced control strategies (Aste, Manfren, and Marenzi, 2017). The reasons are several, from the high cost of design and deploy (Sturzenegger et al., 2016) to the lack of know how transfer between HVAC designers and the academia developing these techniques.

Building IoT related hardware and cloud services are getting cheaper, faster and more reliable, allowing to gather the necessary data to run intelligent BEMS.
Furthermore, several open source libraries coupled with optimization packages (Wetter et al., 2014; Jorissen et al., 2018; Jorissen, Boydens, and Helsen, 2018; Blum and Wetter, 2017) have been developed to improve the knowledge gap between industry and academia. The bottleneck remains the person time required to model and setup a BEMS for a specific building and heating system. This work, carried out within project TEPORE, has the goal to reduce the BEMS setup time by giving to the heating system provider the possibility to remotely update the parameters after the first monitoring phase. TEPORE is the Italian acronym for TErmoregolazione Partecipata e Organizzata per il Residenziale Evoluto; in English: human centric cloud-based heating system control for cutting-edge residential building. It is a project founded by Regione Lombardia in the framework of Smart Living call.

The showcase for this approach is a multi-family residential building located in Milan, North Italy, where the Space Heating (SH) and Domestic Hot Water (DHW) are provided by centralized natural gas boiler (GB) and electric vapour compression heat pump (HP). The problem is divided between estimation of the energy needs and the optimal control of the generation system. To estimate the building thermal load the Modelica Buildings library thermal zone model (Wetter et al., 2014) was coupled with a Model Predictive Control (MPC) algorithm to regulate the thermal zone and achieve thermal comfort. In this work the thermal comfort verification included in the MPC cost function is simplified and intended as matching the room temperature with the thermostat set-point temperature. Then, a model of each component of the heating system was derived starting from datasheets and physical models, designing a Nonlinear MPC (NMPC). The resulting problem was converted into a Constrained Nonlinear Programming (CNLP) optimization problem using the Python library Pyomo (Hart, Watson, and Woodruff, 2011), which optimizes to the required thermal load by shifting between using the HP and the GB. To evaluate the NMPC performance a reference Rule Based Controller (RBC) properly tuned was developed. 


\section{Methodology}

The simulation and optimal control problem were split between estimation of the energy needs and the optimal control of the generation system. Thus, the problem can be divided between a large linear or quadratic optimization problem for the estimation and a smaller Constrained Nonlinear Programming (CNLP) problem for the generation system. The drawback of this approach is that the building envelope thermal inertia cannot be exploited by the optimization as energy storage.

In Figure 1 a simplified scheme of the centralized heating system for the case study is shown.

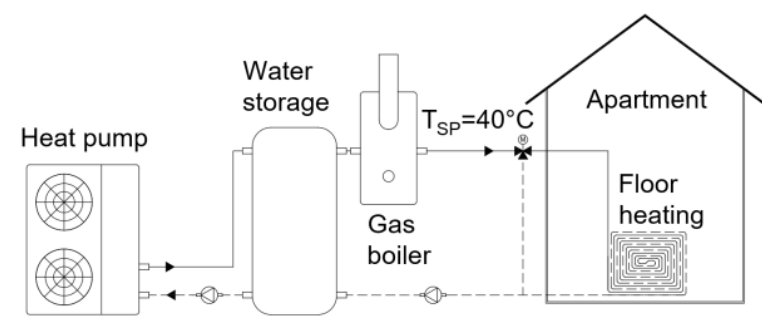

Figure 1: Hybrid heating system layout.

It has a centralized HP with a heating nominal capacity of $24 \mathrm{~kW}_{\text {th }}$ connected to a 5001 water thermal storage, which is in series with a GB with a heating nominal capacity of $114 \mathrm{~kW}_{\text {th }}$ connected to the floor heating system of each apartment.

\section{Building energy needs assessment}

The assessment of the building energy needs was done by simulating a single thermal zone. Afterwards, the entire building energy needs were derived in a simplified way. However, this simplification does not invalidate the results obtained on the generation system, since they are mainly influenced by the fact that the energy need profile is given, rather than slight variations in its shape. The climate data used for the simulations are taken from the typical year built for the Linate weather station in Milano (Energy Plus Weather data).

The model of the thermal zone is developed in Modelica using the "buildings" library (Wetter et al., 2014). The model reproduces a room with mixed air. The boundaries are either connected to the outside or simulate neighbouring thermal zones.

The heating in the room is provided by floor heating. They are connected to a hydraulic circuit composed of a threeway valve to allow recirculation and a pump with constant speed. A constant source with fixed fluid temperature and pressure is located upstream. The opening of the valve and the activation of the pump are both controlled by a PID with a hysteresis cycle to track an optimized set-point for room air temperature.

A trajectory of desired set-point is fed into an MPC optimizer. Once per hour, the optimization anticipates the system evolution of the thermal needs in function of the weather forecasts and the set point profile over the next 6 hours and adjusts the set-point sent to the PID. The evolution forecast is based on a simplified ResistanceCapacity (RC) model of the room. It features the autoregressive dynamics of temperature evolution of the room interiors and of the effective thermal capacity of the floor heating and the effects of the external temperature and heating source.

An open-loop adaptive strategy is designed to deploy the MPC optimizer in different thermal zones. First, a cloudbased solution gathers the input and output of the MPC optimization. Then, the system manager evaluates the distance between MPC predictions and actual temperatures, and finally, can repeat the model calibration and overwrite the parameters used on field, in Figure 2 a simple schematic of the communication protocol is shown.

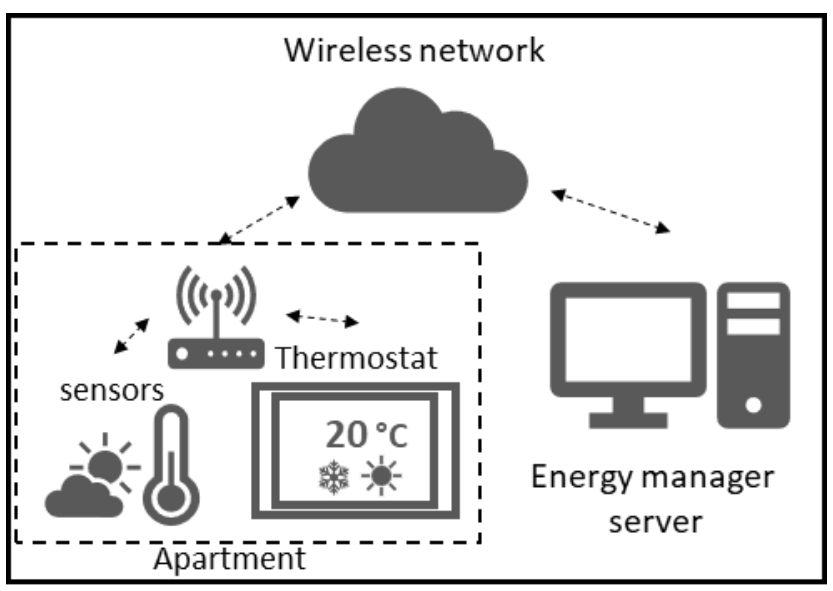

Figure 2: Simple schematic of the communication protocol.

The overall model is run for 120 days from January to April, which are representative of the complete heating season. There are similarities between the cold months December and January, as well as between the average cold months November and February and the beginning and end of the heating season (October against March, April). Starting from the typical year hourly data these were spline interpolated to a time step of 1 minute to catch the useful dynamics for the HVAC and to obtain the heating energy need of a single apartment under realistic use conditions. The interpolation of weather data to such a small time step is reasonable for the external temperature, which tends to have a gradual variation during the day, while radiation is usually recorded as an integral value over the hour and it may not have a gradual variation during the day, but thanks to the relative high capacity of the building it would not impact significantly on the building heat transfers dynamics.

\section{Hybrid heating system modelling}

The first step for the control optimization of the centralized heating system is the modelling of each component (Figure 1), which was done starting from the case study technical schemes and manufacturer's datasheets. 


\section{Storage model}

The thermal storage was modelled using a multi-node stratified approach (Kleinbach, 1990), where each node represents a control volume of water. Since the mass flow rates as inlet and outlet are relatively high, nominal HP flow rate is between 4000 to $8000(\mathrm{l} / \mathrm{h})$ and nominal outlet flow rate can be up to $8000(\mathrm{l} / \mathrm{h})$, while the storage volume is 500 (1); the storage can be considered almost well mixed and three nodes were chosen as representative of the real temperature profile. In Figure 3 a scheme of the storage is presented.

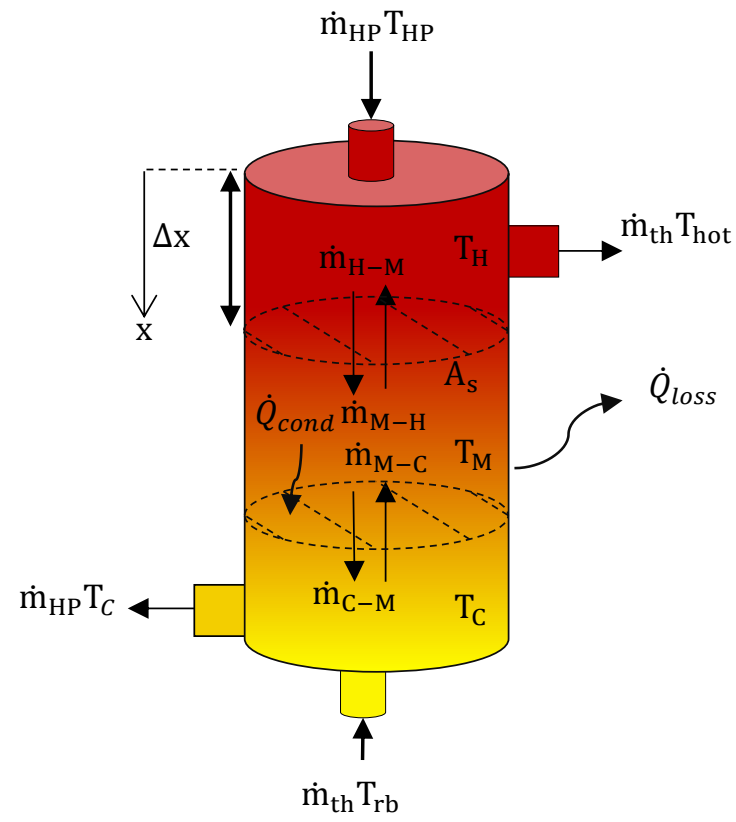

Figure 3: Thermal storage scheme.

$T_{H}, T_{M}$ and $T_{C}\left({ }^{\circ} \mathrm{C}\right), C_{H}, C_{M}$ and $C_{C}(\mathrm{~kJ})$ are respectively, the temperature and capacity of the hot, medium. cold nodes node, while $\dot{m}_{i-j}(\mathrm{~kg} / \mathrm{s})$ are the water mass flow rate between the nodes to account for flow inversion switching from charge to discharge. $\dot{m}_{\mathrm{HP}}(\mathrm{kg} / \mathrm{s})$ is the heat pump flow rate in the thermal storage, while $\mathrm{T}_{H P}\left({ }^{\circ} \mathrm{C}\right)$ is calculated using $\dot{Q}_{H P, n o m}(\mathrm{~kW})$ and $f_{\mathrm{HP}}(-)$, which are the respective inlet power and load factor according to $\mathrm{T}_{\mathrm{HP}}=$ $f_{H P} \dot{Q}_{H P, n o m} / \dot{m}_{\mathrm{HP}} c_{w}-T_{C}$.

$\dot{m}_{t h}(\mathrm{~kg} / \mathrm{s})$ is the mass flow rate from the thermal storage to the building, while $T_{r b}\left({ }^{\circ} \mathrm{C}\right)$ is the return temperature from the building floor heating systems.

$\dot{Q}_{\text {loss }}(\mathrm{kW})$ (1) is the heat rate loss towards the environment. It is calculated as a global heat transfer coefficient $\mathrm{U}\left(\mathrm{kW} / \mathrm{m}^{2} / \mathrm{K}\right)$ multiplied by the external area $A_{x}\left(\mathrm{~m}^{2}\right)$ of each node and the temperature difference between the node and the external temperature considered as constant at $15{ }^{\circ} \mathrm{C}$. $\dot{Q}_{\text {cond }}(\mathrm{kW})(2)$ is the conductive heat transfer between the nodes, which is the temperature difference between the nodes divided by the distance between the nodes $\Delta x(\mathrm{~m})$ and multiplied by the crosssectional $A_{s}\left(\mathrm{~m}^{2}\right)$ and water thermal conductivity $k$ $(\mathrm{kW} / \mathrm{m} / \mathrm{K})$. The resulting dynamic equations for the nodes energy balances are reported in (3-5).

$$
\begin{gathered}
\dot{Q}_{\text {loss }}=U A_{x}\left(T_{\text {ext }}-T_{i}\right) \\
\dot{Q}_{\text {cond }}=\frac{k}{\Delta x} A_{s}\left(T_{i}-T_{j}\right) \\
C_{H} \frac{d T_{H}}{d t}=f_{h p} \dot{Q}_{h p}-\dot{m}_{t h} c_{W} T_{h p}+\dot{Q}_{c o n d}+ \\
\dot{Q}_{\text {loss }}-\dot{m}_{H-M} c_{W}\left(T_{H}\left(1-f_{t h}\right)+T_{M} f_{t h}\right) \\
C_{M} \frac{d T_{M}}{d t}=\dot{m}_{M-H} c_{W}\left(T_{M} f_{\text {th }}+T_{H}\left(1-f_{\text {th }}\right)\right)- \\
\dot{m}_{M-C} c_{W}\left(T_{M}\left(1-f_{t h}\right)+T_{C} f_{t h}\right)+\dot{Q}_{\text {cond }}+\dot{Q}_{\text {loss }} \\
C_{C} \frac{d T_{C}}{d t}=\dot{m}_{t h} c_{W} T_{r b}-\dot{m}_{h p} c_{W} T_{C}+\dot{Q}_{\text {cond }}+\dot{Q}_{\text {loss }}+ \\
\dot{m}_{C-M} c_{W}\left(T_{C}\left(1-f_{t h}\right)+T_{M} f_{t h}\right)
\end{gathered}
$$

The last parameter to consider in the nodes equations is $f_{\text {th }}$, described by (6), that determines the direction of the water flow depending on the mass flow rate balance in the water tank. To describe this behavior without using a bilinear function a hyperbolic tangent shape function has been adopted.

$$
f_{t h}=\frac{1+\tanh \left(\mathrm{L} \dot{m}_{i-j}\right)}{2}
$$

$L(\mathrm{~s} / \mathrm{kg})$ is a constant to emulate a step function and in this way $f_{t h}(-)$ is 1 when the storage is discharging, flow going up, and 0 when the storage is discharging, flow going down.

\section{Air source heat pump and boiler models}

Starting from data available in the datasheet, a model based on performance maps was developed for the air-towater $24 \mathrm{~kW}_{\text {th }}$ HP. The maps contain the values of rated heating power $\dot{Q}_{H P}(\mathrm{~kW})$ transferred to the fluid and coefficient of performance COP in function of the fluid outlet temperature $\mathrm{T}_{\mathrm{HP} \text {,out }}\left({ }^{\circ} \mathrm{C}\right)$, external air temperature and partial load. However, the dependencies of $\dot{Q}_{H P}$ and COP from outlet temperature and load factor were neglected under the assumption that the temperature working range for the HP is restricted between 30 and 40 ${ }^{\circ} \mathrm{C}$ and the load factor rarely goes below $50 \%$ during the simulations. Therefore, the worst-case scenario was assumed for the interpolating polynomials, namely $40{ }^{\circ} \mathrm{C}$ $\mathrm{T}_{\mathrm{HP} \text {,out }}$ and full load operation. The equations are reported below:

$$
\begin{gathered}
\dot{Q}_{H P}=a_{1} T_{\text {ext }}+a_{0} \\
C O P_{H P}=a_{3} T_{e x t}^{3}+a_{2} T_{\text {ext }}^{2}+a_{1} T_{\text {ext }}+a_{0}
\end{gathered}
$$

The coefficients values are listed in Table 1 .

Table 1: HP coefficients.

\begin{tabular}{|c|c|c|}
\hline Coefficients & $\dot{\mathbf{Q}}_{\mathbf{H P}}$ & $\mathbf{C O P}_{\mathbf{H P}}$ \\
\hline$a_{0}$ & $6.66 E-01$ & $-3.51 E-04$ \\
\hline$a_{1}$ & $2.10 E+01$ & $4.96 E-03$ \\
\hline$a_{2}$ & $/$ & $1.36 E-01$ \\
\hline$a_{3}$ & $/$ & $3.37 E+00$ \\
\hline
\end{tabular}


The boiler model was also simplified for the optimization problem assuming a constant efficiency eta on the gross heating factor (GHV) of $90 \%$.

Furthermore, piping losses were neglected considering that the temperature set-point and flow rates are the same between the MPC and RBC.

\section{Generation system control}

To best evaluate the performance of the NMPC a reference control system is needed. Unfortunately, the monitored results of the case-study are underwhelming, due to mismanagement of the hybrid heating system. Therefore, to have a fair comparison the optimized results were compared against an ideal $\mathrm{RBC}$, which tries to keep the storage at constant temperature of $40{ }^{\circ} \mathrm{C}$ and the GB that instantly compensates for the temperature difference between the top node of the storage and the set-point allowing a perfect tracking of the set-point. The same demand profiles and external conditions as for the optimization problem were used. In Figure 4 a high-level schematic of the MPC and RBC control systems workflow is presented. The workflow goes from left to right, starting from the forecasts of the weather, the estimated energy needs and the energy prices, which are used as inputs in the dynamic equations in the plant model and then the plant is controlled by the NMPC or the RBC.

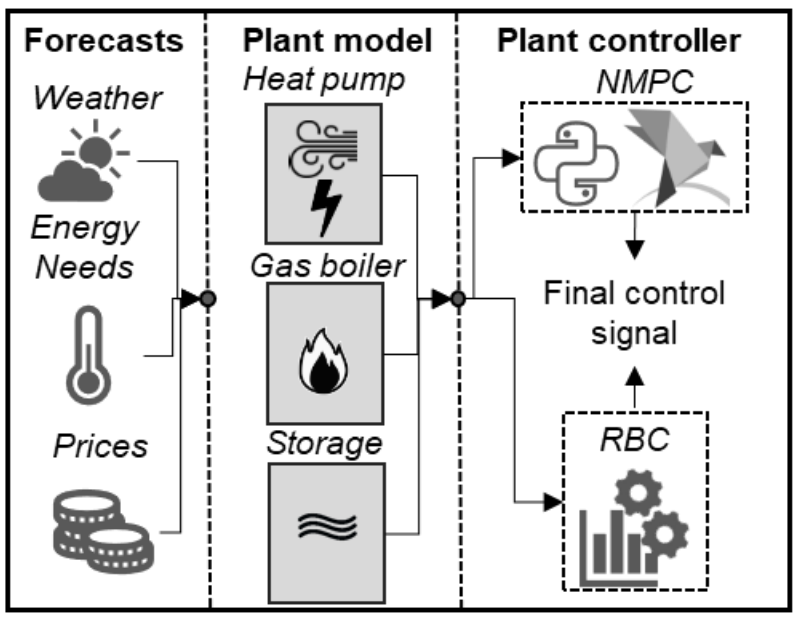

Figure 4: Simulation and optimization scheme for the heating system.

\section{Optimal control problem formulation}

the optimal control problem can be formulated as a cost function that has to be minimized changing the manipulated variables $\mathrm{u}(\mathrm{t})$ constrained by the dynamic state and algebraic equations governing the system. The summary of states $x(t)$, controls $u(t)$, disturbances $w(t)$, which are time dependant non manipulated variables, cost, time horizon and algebraic constraints present in the control problem are presented in Table 2.

Table 2: CLNP formulation.

\begin{tabular}{c|c}
\hline \hline Cost & $\begin{array}{c}\text { Energy consumption }(€) \\
\text { Temperature mismatch }\left(\mathrm{K}^{2} \mathrm{~h}\right)\end{array}$ \\
\hline Stage & $\begin{array}{c}\text { Three (days) per month from Jan } \\
\text { to April. 6,12 and } 72(\mathrm{~h}) \text { as time } \\
\text { horizon. }\end{array}$ \\
\hline Control (u) & $\begin{array}{c}f_{H P}(\mathrm{~kg} / \mathrm{s}):[0,1] \\
\Delta T_{G B}\left({ }^{\circ} \mathrm{C}\right):\left[0, \Delta T_{G B_{m a x}}\right]\end{array}$ \\
\hline Sate (x) & $\left.\begin{array}{c}T_{H}\left({ }^{\circ} \mathrm{C}\right):[-\mathrm{inf}, 45] \\
T_{C}\left({ }^{\circ} \mathrm{C}\right):[-\mathrm{C}):[-\mathrm{inf}, 45]\end{array}\right]$ \\
\hline Disturbances $(\mathbf{w})$ & $\begin{array}{c}T_{\text {ext }}\left({ }^{\circ} \mathrm{C}\right) ; \dot{Q}_{H P n}(\mathrm{~kW}) ; C O P(-) ; \\
\dot{Q}_{\text {need }}(\mathrm{kW}) ; \dot{m}_{t h}(\mathrm{~kg} / \mathrm{s})\end{array}$ \\
\hline Subject to & $\dot{m}_{t h} c_{w}\left(\frac{\mathrm{kJ}}{\mathrm{kgK}}\right) \Delta T_{G B} \leq 114 \mathrm{~kW}$ \\
\hline \hline
\end{tabular}

As shown in Table 2 the objective is to minimize the economic cost of energy provided to the heating system, while guaranteeing the set-point temperature required. A detailed discussion on how to properly formulate the cost function is provided in (Verhelst et al., 2012). The resulting cost function for this case is reported in (9).

$$
\min J_{t o t}(t)=\int_{t_{0}}^{t_{f}}\left(\mathrm{~kJ}_{e n}(t)+(1-k) J_{m i s}(t)\right) d t
$$

$J_{t o t}(t)$ has two contributions, the cost function accounting for energy consumption $J_{e n}(t)$ explained in (10) and the cost function accounting for set-point mismatching $J_{\text {mis }}(t)$ explained in (11). $J_{e n}(t)$ and $J_{m i s}(t)$ are multiplied by $k$, which is a dimensionless parameter that expresses the relative importance between the energy and mismatch cost functions. Thus, for $k=1$, the objective function becomes trivial reducing consumption to zero, while for $k=0$ only the mismatch would be minimized.

$$
J_{e n}(t)=\frac{p_{e l} f_{H P} \dot{Q}_{H P n}(t)}{\operatorname{COP}\left(T_{e x t}\right)}+\frac{p_{n g} \cdot \dot{m}_{t h}(t) c_{w} \cdot \Delta T_{G B}(t)}{\eta_{b}}
$$

The first term represents the cost of electricity to run the $\mathrm{HP}$, where the ratio between the nominal heating capacity of the HP $\dot{Q}_{H P, \text { nom }}$, the load factor $\left(f_{H P}\right)$ and the COP gives the electrical consumption of the HP multiplied by the price of electricity $\left(p_{e l}\right)$. The second term is the gas consumption of the GB multiplied by the average price of natural gas. The reference of both prices is the Italian grid manager GSE (GSE 2017).

$$
J_{m i s}(t)=W \varphi_{\text {on }}(t)\left(T_{\text {supply }}(t)-T_{\text {ref }}\right)^{2}
$$

$T_{\text {supply }}$ is the supply temperature calculated as the top node temperature of the storage plus the GB $\Delta T_{G B}\left({ }^{\circ} \mathrm{C}\right)$, $T_{\text {ref }}=40^{\circ} \mathrm{C}$ is the set-point temperature of the heating system, $\varphi_{o n}(t)$ is 1 when there is demand from the building and 0 otherwise. $W$ is a constant weight that converts the units of the mismatch $\mathrm{K}^{2}$ in price units $€$. 


\section{Numerical approach for optimal control problem}

To solve optimal control problem, it was converted through direct collocation method into a CNLP, which was solved using Interior Point OPTimizer (IPOPT) (Wächter and Biegler 2004) with the help of a Python interface called Pyomo (Hart, Watson, and Woodruff 2011). Different time steps namely $1 \mathrm{~min}, 2 \mathrm{~min}$ and 5 min and the resulting in 2 min being minimum time-step required to catch the storage dynamics.

In Table 3 the values of $J_{e n}$ and $J_{m i s}$ for one value of $k$ and all the prediction horizons are shown.

\section{Table 3: CLNP solution for different time horizons.}

\begin{tabular}{|c|c|c|c|c|c|c|}
\hline \multirow{2}{*}{$\begin{array}{c}\text { prediction } \\
\text { horizon }\end{array}$} & \multicolumn{2}{|c|}{$6 \mathrm{~h}$} & \multicolumn{2}{|c|}{$12 \mathrm{~h}$} & \multicolumn{2}{|c|}{$72 \mathrm{~h}$} \\
\hline & $\begin{array}{c}\mathrm{J}_{\mathrm{en}} \\
€\end{array}$ & $\begin{array}{c}\mathrm{J}_{\mathrm{mis}} \\
\mathrm{K}^{2} \\
\end{array}$ & $\begin{array}{c}\mathrm{J}_{\mathrm{en}} \\
€\end{array}$ & $\begin{array}{c}\mathbf{J}_{\text {mis }} \\
\mathrm{K}^{2} \\
\end{array}$ & $\begin{array}{c}\mathrm{J}_{\mathrm{en}} \\
€\end{array}$ & $\begin{array}{l}\mathbf{J}_{\text {mis }} \\
\mathrm{K}^{2} \\
\end{array}$ \\
\hline Jan & 225 & 0.274 & 220 & 0.274 & 214 & 0.274 \\
\hline Feb & 206 & 0.273 & 202 & 0.273 & 201 & 0.273 \\
\hline Mar & 203 & 0.255 & 197 & 0.254 & 192 & 0.254 \\
\hline Apr & 120 & 0.185 & 108 & 0.184 & 108 & 0.184 \\
\hline
\end{tabular}

The results reported on the table show that increasing the prediction horizon of the MPC even if considered perfect and deterministic, does not bring significant benefit. In fact, $\mathrm{J}_{e n}$ changes by $5 \%$ switching from $6 \mathrm{~h}$ to $72 \mathrm{~h}$, while $\mathrm{J}_{m i s}$ does not have significant variation. This comes from the fact that the thermal storage has a very small capacity compared to the thermal load and the building thermal inertia is not taken into consideration during the optimization process. Therefore, all the following results will be based on the $6 \mathrm{~h}$ prediction horizon.

\section{Results}

\section{Overview on economic and energetic performance}

In this section, the results for the typical days of the simulated months are reported in terms of energy bills and energetic performance comparing the results of the MPC and the RBC.

In Figure 5 the solutions from January to April of the RBC (grey dots) are compared to the NMPC solutions (colored dots) varying the parameter $k$ (the considered value is near the corresponding dot on the figure). The energy cost is plotted on the $\mathrm{x}$-axis against $\Delta T_{m i s}$. The latter is the cumulative difference between the supply temperature and its reference $\mathrm{T}_{\text {ref }}=40{ }^{\circ} \mathrm{C}$ evaluated only during the operating hours as shown in (12).

$$
\Delta T_{\text {mis }}=\frac{\sqrt{\int_{t_{0}}^{t_{f} J_{m i s}(t)}}}{h_{o p}} \quad{ }^{\circ} \mathrm{C}
$$

Thus, $\Delta T_{\text {mis }}$ is the hourly average difference between $T_{\text {supply }}$ and $T_{\text {ref }}$ during operating hours. When this value is between zero and one, $0 \leq \Delta T_{\text {mis }} \leq 1$, the supply temperature will be, $39 \leq T_{\text {supply }} \leq 41{ }^{\circ} \mathrm{C}$.

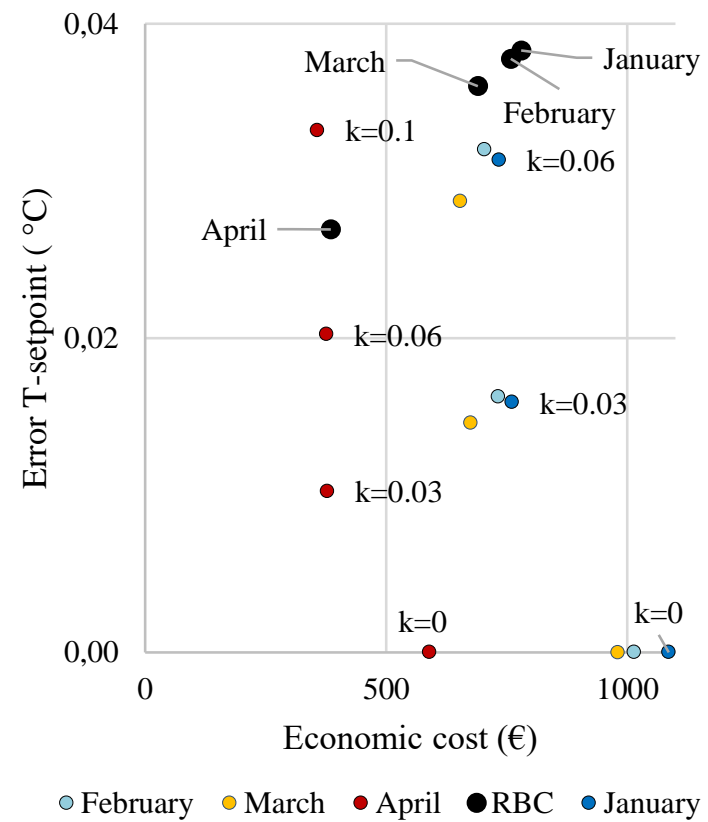

Figure 5: monthly results NMPC vs RBC in terms of energy cost and set-point mismatch for different $k$ values (dots).

Increasing the value of $k$, increases the economic weight while reducing the mismatch weight in the cost function. Since $k$ 's trend is hyperbolic as for Pareto optimization problems, after a certain threshold reducing its value will only increase the temperature mismatch without reducing the energy cost. Taking $\Delta T_{m i s}=0.04{ }^{\circ} \mathrm{C}$ as the limit value for mismatch the corresponding $k$ value solution has been considered as the optimum of the CLNP. However, it can be noted that solutions with $\mathrm{k}$ higher than 0 are all aligned, meaning that by changing the value of $k$, the economic cost will remain similar, while the mismatch error will increase. This is the first hint that there might be much room for the optimization of this problem, in fact looking at Figure 6, where the economic results, in terms of energy bills, achieved by the best solution of the MPC and $\mathrm{RBC}$ are plot against each other

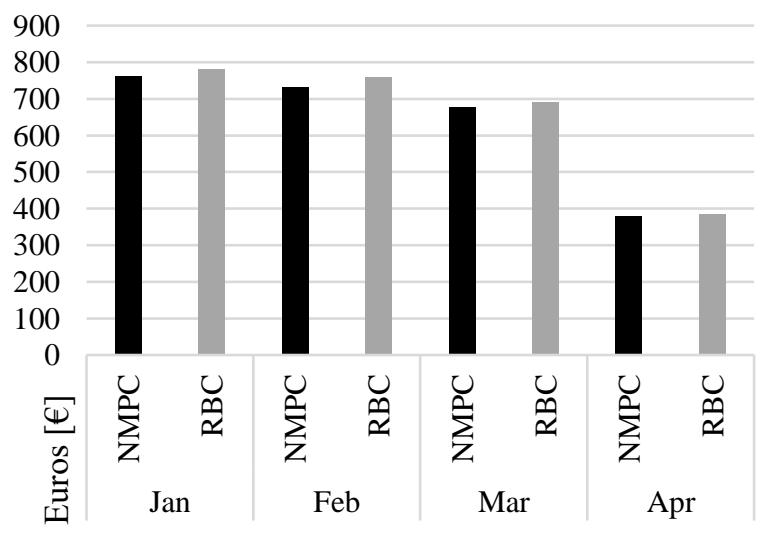


Figure 6: monthly energy cost for space heating:

NMPCs vs RBC.

The resulting economic savings from the NMPC are underwhelming due to the current heating system setup, the NMPC does not have enough degrees of freedom to significantly improve the operation of the hybrid heating system. In particular, the HP heating capacity is too small and almost always works already at full load in both cases, and the storage is not big enough to guarantee more than few minutes at nominal flow rate required by the building. To further prove this point in Figure 7 is shown the total distribution of heat provided respectively by HP and GB for all the months comparing the MPC best solution and RBC simulations.

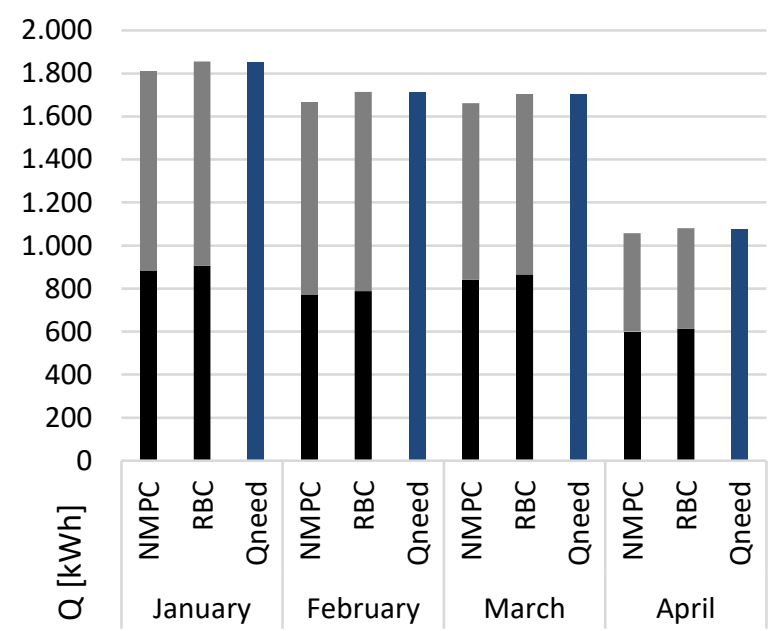

Figure 7 thermal energy generated by HP (black) and $G B$ (grey), beside the $Q_{\text {need }}$ required (blue).

This chart shows how the relative percentage of thermal energy generated is similar for the NMPC and the RBC.

The second interesting thing to notice is that the heat contribution from the NMPC is slightly lower with respect to the $\mathrm{RBC}$, this is due to the fact that at the beginning of the simulation the storage was initialized at $40{ }^{\circ} \mathrm{C}$ and at the end of the simulation the NMPC leaves it at a temperature on average below $30^{\circ} \mathrm{C}$, while the $\mathrm{RBC}$ is tuned to always charge it back. However, this was accounted for in the previously presented in Figure 6.

\section{HP and GB profiles comparison for typical day in January and April}

Another way to check that the NMPC and the RBC have a similar behaviour is to compare the HP power and GB thermal heat rate. January (Figure 8) and April (Figure 9) were chosen as example

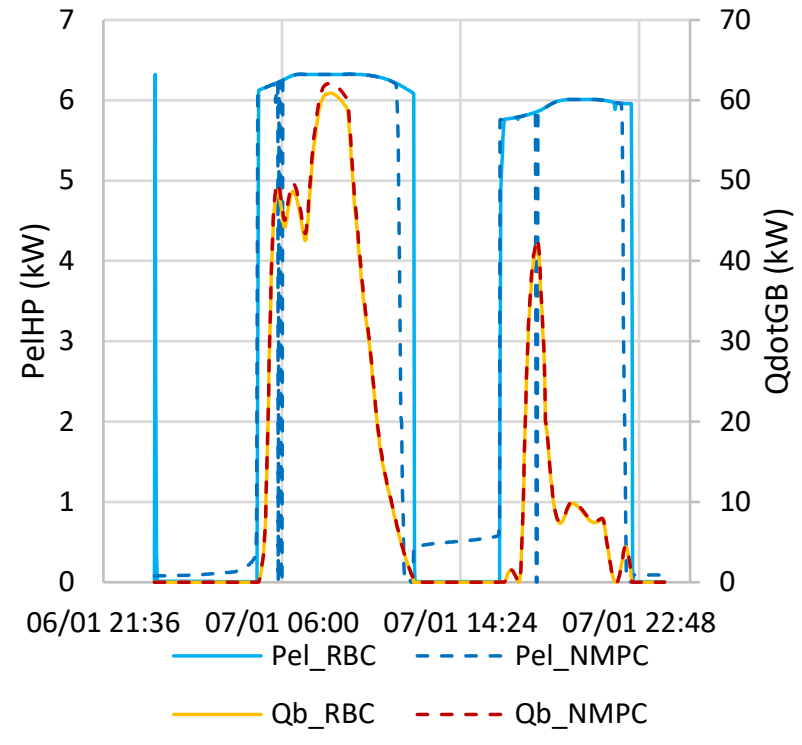

Figure 8: a typical day of January. On the x-axis the date and time. On the left y-axis the electrical power of $H P$ and on the right y-axis the heat rate of the GB. $N M P C$ results (solid lines) vs $R B C$ results (dashed lines).

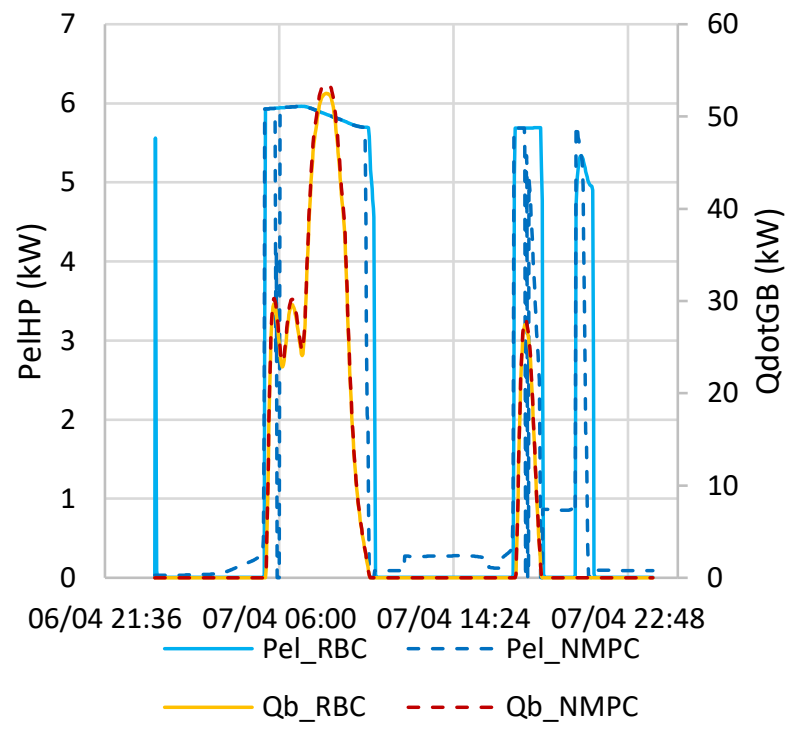

Figure 9: a typical day of April. On the $x$-axis the date and time. On the left y-axis the electrical power of HP and on the right $y$-axis the heat rate of the GB. NMPC results (solid lines) vs RBC results (dashed lines).

Both in the January and April case, the NMPC and RBC have a very similar behaviour, the only notable difference is that the NMPC tries to use the HP at partial load to recharge the storage while there is no $Q_{\text {need }}$, because those are the central hours of the day with a higher Text and therefore a higher $C O P$ of the HP. Furthermore, the average temperature of the thermal storage is lower reducing its heat losses. 


\section{Simplified design analysis of the HP and GB}

In this study was proven that the benefit of a NMPC with respect to a well-tuned RBC is not significant (2-3\%) for this specific case study, due to the small thermal capacity of the HP and the storage, and the incapability to exploit the inertia of the building since the CNLP just tracks the given $Q_{\text {need }}$ profile.

The same optimization framework was also used to investigate how the capacity of the heat pump affects the performance of the NMPC by doubling the capacity of the HP to $48 \mathrm{~kW}_{\text {th }}(+100 \%)$ and reducing the GB capacity to $90 \mathrm{~kW}_{\text {th }}(-21 \%)$, the storage size was not increased because the authors did not want to increase the degree of freedom for the CNLP. Comparing the performance of the new NMPC called NMPChp+ and the ideal RBChp+ with the same change in HP and GB capacities, there was no significant difference with respect to the previous case, for the same reasons mentioned above.

However, an interesting insight was obtained by the comparison of the simulation with the baseline HP and GB (NMPC) capacity and the new one with the increased capacity of the HP (NMPChp+). The energy bills for NMPChp+ decrease by almost $50 \%$ with respect to the NMPC case as shown in Figure 10.

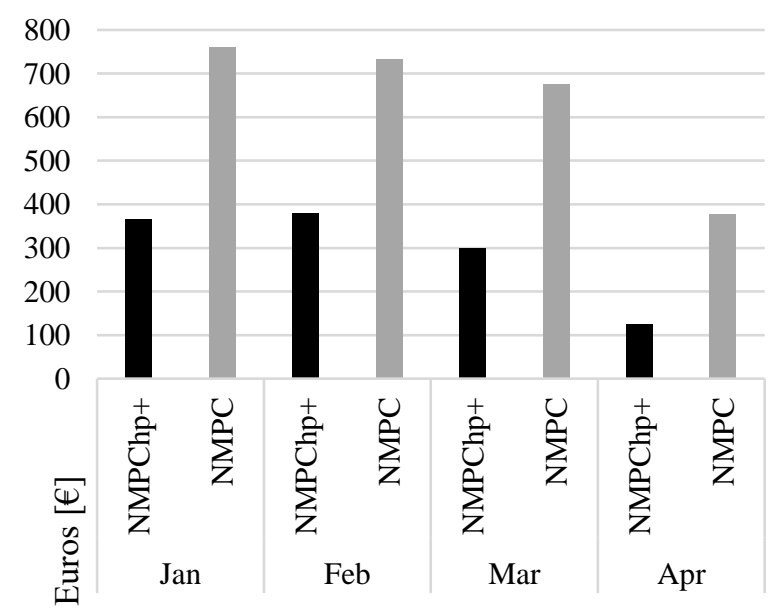

Figure 10 Monthly energy cost NMPChp+ (black) vs NMPC (grey)

The reason behind this improvement on the energy bills cost, on average higher than $50 \%$ is due to the increase in share of heat produced by the HP as it can be seen in Figure 11 meaning that the decrease in cost of the energy bills is linear with respect to the heat pump size. However, this holds true until the HP size is small enough to work almost always between 60 and $100 \%$ of its nominal capacity. If the heat pump starts working outside this range the COP will drastically drop and the HP will undergo a series of on-off cycle which will harm the HP efficiency and durability.

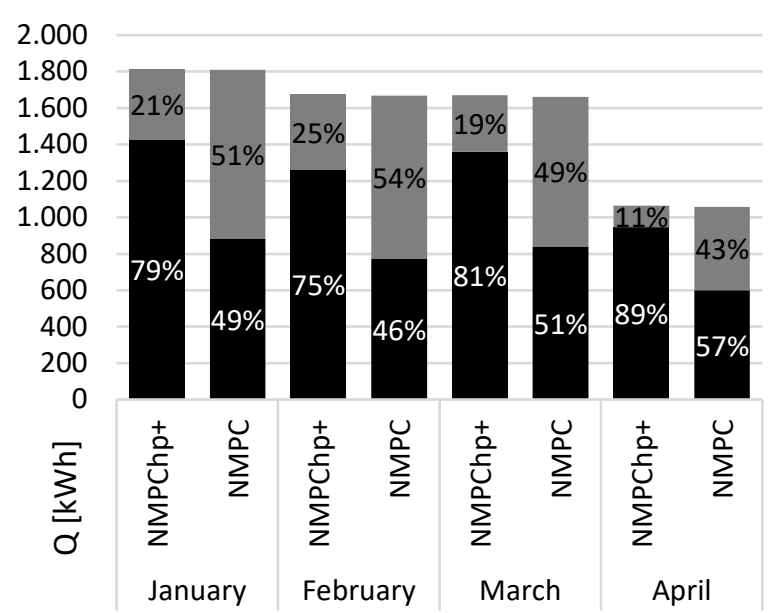

Figure 11 Cumulative heat provided divided between HP (black) and GB (grey) comparing the NMPChp+vs NMPC

Obviously, these results are biased towards the specific HP and GB chosen, the climatic condition, especially because the HP defrosting cycle was neglected, the energy prices of electricity and natural gas, and the higher investment cost of the HP. However, they stress the importance of coupling the optimization in the design phase with optimal control to achieve the best economic and energetic performance.

\section{Conclusions}

This study presented a North Italian case study within the project TEPORE. The main insights from this work are:

i) The improvement of comfort performance, intended as the average room temperature reaching the thermostat setpoint temperature, when using an MPC algorithm with respect to a traditional approach.

ii) The underwhelming improvement in reducing the hybrid heating system running costs when using a NMPC compared to an ideal RBC, due to the small size of the HP and the storage, and the incapability to exploit the inertia of the building since the CNLP just tracks the given $Q_{\text {need }}$ profile. This demonstrates that using an MPC to achieve comfort and a properly tuned RBC are more than enough to have a good performance of this simple system. This is especially true for new buildings where the energy consumption is already low and achieving comfort with a traditional approach may be tricky, because of the long building fabric inertia.

iii) When considering the variation on the size of the HP and the GB, more specifically by doubling the HP capacity from 24 to $48 \mathrm{~kW}_{\text {th }}(+100 \%)$ and reducing the capacity of the boiler from 114 to $90 \mathrm{~kW}_{\mathrm{th}}(-21 \%)$, the running costs reduce on average by around $50 \%$. Even though these results are biased towards the specific HP and GB chosen, the climatic condition, the energy prices of electricity and natural gas, and the higher investment cost, they stress the importance of coupling the optimization in the design phase with optimal control to 
achieve the best economic and energetic performance. This is especially true when considering the EU goal of reducing building energy footprint, that can be achieved only by introducing more complex solutions that includes large storages and the introduction of renewable energies. Therefore, future studies will be carried out in developing a combined system design and control optimization tool.

\section{Acknowledgments}

The authors would like to thank Regione Lombardia for funding of the TEPORE project (project ID 379389) and Emanuele Mason from ENERSEM for his contribution in the space heating energy need estimation and MPC.

\section{References}

Afram, A., and Farrokh J. (2014). "Theory and Applications of HVAC Control Systems - A Review of Model Predictive Control (MPC)." Building and Environment.

Aste, N., Manfren, M., and Marenzi, G. (2017). "Building Automation and Control Systems and Performance Optimization: A Framework for Analysis." Renewable and Sustainable Energy Reviews 75 313-30.

Blum, David H, \& Michael Wetter. (2017). "MPCPy: An Open-Source Software Platform for Model Predictive Control in Buildings." Proceedings of the 15th IBPSA Conference, 1381-90.

Coninck, R, and Helsen, L. (2016). "Practical Implementation and Evaluation of Model Predictive Control for an Office Building in Brussels.” Energy and Buildings 111: 290-98.

Energy Plus Weather data. n.d. "Energy Plus Weather Data." https://energyplus.net/weather.

Eurostat. (2016). "Eurostat:Consumption of Energy.". http://ec.europa.eu/eurostat/statisticsexplained/index.php/Consumption_of_energy.

GSE. (2017). "Gestore Servizi Energetici.”. https://www.gse.it/en.
Hart, W. E., Watson J. P., and Woodruff. D. L. (2011). "Pyomo: Modeling and Solving Mathematical Programs in Python." Mathematical Programming Computation 3 (3): 219-60.

Jorissen, F., W. Boydens, and L. Helsen. (2018). "TACO, an Automated Toolchain for Model Predictive Control of Building Systems: Implementation and Verification." Journal of Building Performance Simulation 1493.

Jorissen, F., G. Reynders, R. Baetens, D. Picard, D. Saelens, and L. Helsen. (2018). "Implementation and Verification of the IDEAS Building Energy Simulation Library." Journal of Building Performance Simulation 1493: 1-20.

Kleinbach, E. M.. (1990). "Performance Study of OneDimensional Models for Stratified Thermal Storage Tank by Master of Science University of Wisconsin-Madison" 50 (2): 155-66.

Sturzenegger, D., D., Gyalistras, M., Morari, and R., S Smith. (2016). "Model Predictive Climate Control of a Swiss Office Building." IEEE Transactions on Control Systems Technology 24 (1): 1-12.

Thieblemont, H., F., Haghighat, Ryozo O., and A., Moreau. (2017). "Predictive Control Strategies Based on Weather Forecast in Buildings with Energy Storage System : A Review of the State-ofthe Art." Energy \& Buildings 153: 485-500.

Verhelst, C., F. Logist, J. V., Impe, and L. Helsen. (2012). "Study of the Optimal Control Problem Formulation for Air-to-Water Heat Pump Systems." KU Leuven, 43-46.

Wächter, A, and L. T. Biegler. (2004). "On the Implementation of a Primal-Dual Interior Point Filter Line Search Algorithm for Large-Scale Nonlinear Programming (IPOPT)." Mathematical Programming, Volume 106, Issue 1, pp 25-57

Wetter, M., W. Zuo, T. S. Nouidui, and X. Pang. (2014). "Modelica Buildings Library." Journal of Building Performance Simulation 7 (4): 253-70. 